\title{
CONTROL IN VITRO E IN VIVO DE GARRAPATAS (Rhipicephalus (Boophilus) microplus) UTILIZANDO LA RAIZ PULVERIZADA DEL BARBASCO (Lonchocarpus nicou (Aublet) DC.)
}

\author{
Yemen MEJIA-AGUIRRE ${ }^{1}$ y Daniel PAREDES-LOPEZ ${ }^{2 *}$ \\ 1 Granja Zootécnica Universidad Nacional Agraria de la Selva Av. Universitaria s/n, PO BOX 156, Tingo María, Perú. \\ 2 Area de Sanidad Animal, Departamento de Ciencia Animal, Universidad Nacional Agraria de la Selva, Av. Universitaria \\ s/n, PO BOX 156, Tingo María, Perú. *e-mail: daniel.paredes9@gmail.com.
}

\section{RESUMEN}

El presente estudio tuvo como objetivo determinar el control in vitro e in vivo de garrapatas (Rhipicephalus (Boophilus) microplus) utilizando la raíz pulverizada del barbasco (Lonchocarpus nicou (Aublet) $D C$.). La raíz fresca se trozó, deshidrató a $50^{\circ} \mathrm{C}$ y se trituró en un molino Willy hasta $1 \mathrm{~mm}$ de partícula. En la prueba in vitro se distribuyeron 150 huevos y 150 larvas en grupos de 10 y se colocaron sobre papel filtro Wattman $\mathrm{N}^{\circ} 40$, embebidos con las suspensiones de $0 ; 0,1 ; 0,25 ; 0,5$ y $0,75 \mathrm{~g} / 100 \mathrm{~mL}$ de la raíz pulverizada de barbasco con tres repeticiones por cada tratamiento. Para el trabajo in vivo se emplearon cinco vaquillas de 1,5 años en promedio, distribuyéndose en 5 tratamientos; 0,00 (T0); 0,10 (T1); 0,25 (T2); 0,50 (T3) y $0,75 \mathrm{~g} / 100 \mathrm{~mL}$ (T4) con 3 repeticiones. Los resultados obtenidos fueron analizados empleando chi cuadrado. In vitro e in vivo, la mortalidad de larvas y adultos respectivamente obtenidos en T2, T3 y T4, fueron mayores al blanco control (T0) $(\mathrm{P}<0,0001)$, a partir de los 15 minutos in vitro y a partir de las 24 horas in vivo. La raíz pulverizada del barbasco redujo la viabilidad larvaria post nacimiento a partir de $0.10 \mathrm{~g} / 100$ $\mathrm{mLl}$ de concentración y en la mortalidad de larvas y garrapatas adultas a partir de $0.50 \mathrm{~g} / 100 \mathrm{~mL}$ de concentración.

PALABRAS CLAVE: Lonchocarpus nicou, Rhipicephalus microplus, efecto biocida, garrapatas.

\section{IN VITRO E IN VIVO CONTROL OF TICKS (Rhipicephalus (Boophilus) microplus) USING BARBASCO (Lonchocarpus nico (Aublet) DC.) PULVERIZED R00T.}

\section{ABSTRACT}

The aim of the present research was to determine the in vitro and in vivo control of ticks (Rhipicephalus (Boophilus) microplus) by dusted barbasco (Lonchocarpus nicou (Aublet) DC. For this purpose the fresh barbasco root was firstly chop up, dehydrated at $50^{\circ} \mathrm{C}$ for 72 hours and milled in a willy mill at $1 \mathrm{~mm}$ size particle. For In vitro test, 150 tick eggs and larvae were distributed in 15 groups of 10 ticks each, then were placed on wattman $\mathrm{N}^{\circ} 40$ filter paper embed in the $0, .0 .1,0.25,0.5$ and $0.75 \mathrm{~g} / 100 \mathrm{~mL}$ suspension of dusted barbasco root with three repetitions for treatment. For in vivo procedure, 5 tick parasitized heifers 1.5 years old were distributed in five treatments 0 (T0), 0.10 (T1), 0.25 (T2), 0.50 (T3), $0.75 \mathrm{~g} / 100 \mathrm{~mL}$ (T4) with three repetitions each were used. Data was analyzed by Chi-square test. In either methods, In vitro and in vivo, larvae and adult mortality obtained in T2, T3, and T4 were higher than those obtained in the T0 $(\mathrm{P}<0.0001)$ after 15 minutes in vitro and 24 hours in vivo. Dusted barbasco root slowed down larval viability post hatch after $0.1 \mathrm{~g} / 100 \mathrm{~mL}$ and in the larvae and adults mortality after $0.5 \mathrm{~g} / 100 \mathrm{~mL}$ concentration.

KEYWORDS: Lonchocarpus nicou, Rhipicephalus microplus, biocidal effect, ticks. 


\section{INTRODUCCIÓN}

Uno de los problemas que afectan a la ganadería peruana es la infección por parásitos externos o ectoparásitos, entre los que se encuentran diferentes especies de garrapatas, lo que influye en la productividad pecuaria además de producir enfermedades secundarias. Desde hace mucho tiempo, la práctica más común para el control de las garrapatas ha sido la utilización de productos químicos. En el desarrollo de la ganadería, se aplican tratamientos que permiten al ganadero prevenir o controlar plagas y enfermedades, sin embargo, los productos que se aplican pueden causar efectos colaterales sobre la salud animal y humana, además de que pueden provocar diversos niveles de contaminación de la carne, la leche y el medio ambiente (Henrioud, 2011).

Debido al uso prolongado de los acaricidas químicos, la garrapata ha desarrollado resistencia a los mismos en todo el mundo, sobre todo en aquellos lugares donde se han utilizado dosis elevadas (Casas, et al., 2009 ). Frente a este problema, hemos recurrido a los conocimientos de los pobladores rurales, quienes han logrado mitigar con un alto porcentaje de éxito este problema, usando plantas medicinales y derivados de las mismas que poseen una buena actividad biocida (Silva, et al., 2002), encontrando, de esta forma, una muy buena alternativa etnobotánica que se podría utilizar para implementar estrategias de control y manejo integrado de garrapatas .

El presente estudio tuvo como objetivo evaluar el control in vitro e in vivo de garrapatas (Rhipicephalus (Boophilus) microplus), usando la raíz pulverizada del barbasco (Lonchocarpus nicou (Aublet) DC.).

\section{MATERIALES Y MÉTODOS}

\section{Obtención de la raíz pulverizada del barbasco Material vegetal}

Las raíces frescas del barbasco (Lonchocarpus nicou (Aublet) DC.) fueron obtenidas en el caserío de Agua Blanca, distrito de Monzón, provincia de Huamalies, en el departamento de Huánuco. La identificación taxonómica de la planta se llevó a cabo con el asesoramiento de los especialistas en botánica de la facultad de Recursos Naturales Renovables de la Universidad Nacional Agraria de la Selva.

Las raíces se picaron, deshidrataron a $50^{\circ} \mathrm{C}$ por 72 horas, se pulverizaron en un molino y finalmente se refrigeraron a $5^{\circ} \mathrm{C}$ hasta su empleo (Mariños et al., 2004).

\section{Preparación de extractos}

Para preparar la suspensión se tomó como dosis de referencia la utilizada por Mariños et al., (2004) de 0,15 a $6,25 \mathrm{~g} / \mathrm{L}$ del polvo de raíz de Lonchocarpus utilis. Se prepararon 4 dosis de $0,10,0,25,0,50 \mathrm{y}$ $0,75 \mathrm{~g}$ de la raíz pulverizada de Lonchocarpus nicou (Aublet) DC., que fueron pesadas en una balanza analítica y diluidas en $100 \mathrm{ml}$ de agua destilada en un vaso de precipitación, homogeneizándose posteriormente y filtrándose por una tela fina (tafena). Cada una de las concentraciones fue probada por triplicado sobre 10 huevos y 10 larvas de Rhipicephalus (Boophilus) microplus por repetición, utilizándose un total de 30 huevos y 30 larvas por dosis y la misma cantidad en el grupo control. En el grupo control se empleó agua destilada.

\section{Prueba in vitro \\ Material Biológico}

Se emplearon 150 larvas y 150 huevos del género Rhipicephalus (Boophilus) microplus, obtenidos de especímenes adultos colectados de ganado vacuno.

Los adultos utilizados fueron previamente lavados y secados y luego colocados en placas Petri para la ovoposición y eclosión de los huevos.

Posteriormente, los 150 huevos y 150 larvas, con diez días de edad viable, se embebieron en papel filtro wattman $\mathrm{N}^{\circ} 40$ con la solución de la raíz pulverizada del barbasco (Lonchocarpus nicou (Aublet) DC.). Se evaluó la respuesta de estímuloreflejo, separando las larvas vivas de las muertas a los 5,10 y 15 minutos.

\section{Prueba in vivo \\ Material biológico}

Se emplearon 5 vaquillas (1,5 años en promedio) de la raza Holstein y Brown swiss, las vaquillas se seleccionaron entre una población de 15 animales de explotación semi-intensiva. Se verificó que las vaquillas estaban infectadas con garrapatas del género Rhipicephalus (Boophilus) microplus. Se identificaron tres áreas con mayor infestación, localizadas en las regiones internas de la pierna (ingle) y en la tabla del cuello por ambos lados.

Se identificaron tres áreas de $100 \mathrm{~cm}^{2} /$ animal con infestación de garrapatas (Rhipicephalus (Boophilus) microplus), realizándose el conteo antes y después de la aplicación mediante la técnica del método del centímetro cuadrado (Bazán, 2002).

La aplicación se realizó con bombas manuales de 20 litros, utilizando una solución de 4 litros por animal. La dosis por bomba fue de $0,10,0,25,0,50 \mathrm{y}$ 
$0,75 \%$ de la raíz pulverizada del barbasco (Lonchocarpus nicou (Aublet) DC.). Los animales fueron completamente asperjados hasta penetrar el pelo del animal. Se realizó una sola aplicación. A las 24, 48 y 72 horas después de haber sido realizada la aplicación, se realizó el conteo de las garrapatas que seguían pegadas en las zonas marcadas y por diferencia obtuvimos las garrapatas que se desprendieron.

\section{Análisis estadístico}

Para determinar el desprendimiento de las garrapatas adultas del animal por efecto de la raíz pulverizada de Lonchocarpus nicou se empleó la prueba de Chi $x^{2}$ cuadrado

\section{RESULTADOS Y DISCUSIÓN}

\section{Viabilidad embrionaria de Rhipicephalus (Boophilus) microplus in vitro}

En la Tabla 1 se muestran los resultados de la viabilidad embrionaria, fluctuando sus valores de 33,33 a 43,33\%, para el T1 y T2; la mortalidad larvaria en los tres tratamientos con inclusión de la raíz pulverizada de Lonchocarpus nicou (Aublet) $D C$ fue de $100,00 \%$, lo que indica que todas las larvas que eclosionaron murieron posiblemente debido a la alta sensibilidad de las mismas al barbasco, tanto dentro del huevo como a los pocos minutos después de la eclosión. El análisis estadístico con chí $x^{2}$ mostró dependencia significativa $(\mathrm{p}<0,0001)$, lo que indica que existe una alta sensibilidad de los huevos y larvas de Rhipicephalus (Boophilus) microplus a la raíz pulverizada de Lonchocarpus nicou (Aublet) DC.

\section{Mortalidad larvaria de Rhipicephalus (Boophilus) microplus in vitro}

Los tratamientos con las concentraciones de 0,50 y $0,75 \mathrm{mg} / 100 \mathrm{uL}$ a los 10 minutos de inmersión, presentaron valores de mortalidad de 90 y $100 \%$ respectivamente. A los 15 minutos, con la concentración de $0,50 \mathrm{~g} / 100 \mathrm{~mL}$ se obtuvo el $100 \%$ de mortalidad. Las dosis de 0,10 y $0,25 \mathrm{~g} / 100 \mathrm{~mL}$ después de 10 minutos de inmersión, presentaron valores de mortalidad de 33 y $73 \%$, siendo los valores a los 15 minutos de 43 y $83 \%$ respectivamente. El análisis estadístico con chí $x^{2}$ mostró dependencia significativa $\mathrm{d}(\mathrm{p}<0.0001)$, lo que indica que existe relación directa entre la concentración de la raíz pulverizada de Lonchocarpus nicou y el tiempo de acción sobre la mortalidad larvaria.

\section{Desprendimiento del estadio adulto de Rhipicehalus (Boophilus) microplus) in vivo}

En el Tabla 3 se observa el porcentaje de desprendimiento de las garrapatas adultas del animal después del baño con la solución de la raíz pulverizada del barbasco (Lonchocarpus nicou), la garrapatosis fue heterogénea entre tratamientos antes de la aplicación. En los animales que fueron bañados con agua, sin adición del barbasco (T0), el desprendimiento de garrapatas osciló de 2,33 a 9,30\% durante el tiempo evaluado (hasta 72 horas), mientras que en los demás tratamientos el desprendimiento fue progresivamente creciente entre tratamientos y tiempos de evaluación. El desprendimiento a las 72 horas con las concentraciones de $0,10,0,25,0,50$ y $0,75 \mathrm{~g} / 100 \mathrm{~mL}$ fue de $83.33,94.23,100.00$ y $100.00 \%$ respectivamente.

Cuando se evaluó la eclosión de los huevos de Rhipicephalus (Boophilus) microplus in vitro en el tratamiento control (T0), utilizando agua destilada, la eclosión fue del $90 \%$, quedando un porcentaje del $10 \%$ sin eclosionar, debido a la infertilidad de los mismos, estando este resultado en concordancia con los estudios realizados por (Gutiérrez, 2006), que reporta altos porcentajes de eclosión, mayores al $80 \%$, en ejemplares normales sin alteraciones morfológicas visibles y que no hayan sufrido en el manipuleo ni estén afectados por algún tratamiento ixodicida o por el excesivo calor, luz solar, etc.

La investigación reporta, en aquellos tratamientos donde se usó barbasco (Lonchocarpus nicou) en concentraciones de $0,10,0,25,0,50$ y 0,75 $\mathrm{g} / 100 \mathrm{~mL}$, una eclosión de 43,33, 33,33, 40,00 y $36,67 \%$ respectivamente, la baja eclosión se debe probablemente al efecto de la rotenona, principal componente antiparasitario del barbasco. Las larvas que eclosionaron murieron en un $100.00 \%$ en todo los tratamientos, debido a que el metabolismo de las mismas es paralizado por el efecto de la rotenona, inhibiendo el transporte de electrones a nivel de mitocondria y bloqueando la fosforilación del ADP a ATP (Reyes, 2011). Por esto se dice que actúa inhibiendo el metabolismo del artrópodo.

En el Tabla 2 se muestra una mortalidad de 43,33, $83,33,100$ y $100 \%$, para las concentraciones de $0,10,0,25,0,50$ y $0,75 \mathrm{~g} / 100 \mathrm{~mL}$ respectivamente, a los 15 minutos después de la prueba de inmersión. Posiblemente estos niveles de mortalidad se deben a la acción de la rotenona, que tiene la propiedad de atacar al sistema nervioso impidiendo el transporte de iones (Mariños et al., 2004).

Los tratamientos en los que se usó el barbasco a las 72 horas post-tratamiento, mostraron del 83,33 
al $100 \%$ de desprendimiento, debido a que la rotenona afecta al sistema nervioso a nivel de la mitocondria, bloqueando la fosforilación del ADP a ATP e inhibiendo el metabolismo del artrópodo, por lo que la garrapata pierde su capacidad de adherencia sobre el animal y por consiguiente cae. Otros resultados, como los obtenidos por Espinoza (1994), reportan que el extracto del barbasco (Lonchocarpus nicou) al 7 y $10 \%$ contra piojos de cuyes (Gyropus ovalis y Gliricola porcelli) produjo una mortalidad de $100 \%$ a los 3 días después de la aplicación, Peralta (2004) reporta que la harina del Kumu (Lonchocarpus $s p$ ) en concentraciones de 10,20 y $30 \mathrm{~g} / \mathrm{L}$ en baños de inmersión contra piojos de cuyes, obtuvo una mortalidad de $100 \%$ a 2 días posttratamiento. Del mismo modo, trabajos realizados usando diferentes microorganismos han reportado resultados promisorios en el control de garrapatas (Espinoza, 2005).

Tabla 1. Mortalidad de huevos y larvas de Rhipicephalus (Boophilus) microplus in vitro al tratamiento con raíz pulverizada de Lonchocarpus nicou Aublet) DC

\begin{tabular}{cccc}
\hline Concentración $(\mathbf{g} / \mathbf{1 0 0} \mathbf{~ m L})$ & $\begin{array}{c}\text { Viabilidad de } \\
\text { huevos }\end{array}$ & $\begin{array}{c}\text { Larvas } \\
\text { muertas }\end{array}$ & $\begin{array}{c}\text { Huevos } \\
\text { evaluadas }\end{array}$ \\
\hline T0 $(0,00)$ & $27(90,00 \%)$ & $2(7,41 \%)$ & 30 \\
T1 $(0,10)$ & $13(43,33 \%)$ & $13(100,00 \%)$ & 30 \\
T2 $(0,25)$ & $10(33,33 \%)$ & $10(100,00 \%)$ & 30 \\
T3 $(0,50)$ & $12(40,00 \%)$ & $12(100,00 \%)$ & 30 \\
T4 $(0,75)$ & $11(36,67 \%)$ & $11(100,00 \%)$ & 30 \\
\hline Chi cuadrado: $p$ - valor & $<0,0001$ & $<0,0001$ & \\
\hline
\end{tabular}

Tabla 2. Mortalidad larvaria de Rhipicephalus (Boophilus) microplus en periodo de $5-15$ minutos por acción de la raíz pulverizada de Lonchocarpus nicou in vitro

\begin{tabular}{ccccc}
\hline Concentración $(\mathbf{g} / \mathbf{1 0 0} \mathbf{~ m L})$ & \multicolumn{3}{c}{ Larvas muertas } & $\begin{array}{c}\text { Larvas } \\
\text { evaluadas }\end{array}$ \\
\cline { 2 - 4 } & $\mathbf{5}$ minutos & $\mathbf{1 0}$ minutos & $\mathbf{1 5}$ minutos & 30 \\
\hline T0 $(0,00)$ & 0 & 0 & 0 & 30 \\
T1 $(0,10)$ & $2(6,67 \%)$ & $10(33,33 \%)$ & $13(43,33 \%)$ & 30 \\
T2 $(0,25)$ & $10(33,33 \%)$ & $22(73,33 \%)$ & $25(83,33 \%)$ & 30 \\
T3 $(0,50)$ & $16(53,33 \%)$ & $27(90,00 \%)$ & $30(100,00 \%)$ & 30 \\
\hline T4 $(0,75)$ & $23(76,67 \%)$ & $30(100,00 \%)$ & $30(100,00 \%)$ & \\
\hline Chi cuadrado: $p$ - valor & $<0,0001$ & $<0,0001$ & $<0,0001$ & \\
\hline
\end{tabular}

Tabla 3. Mortalidad de adultos de (Rhipicehalus (Boophilus) microplus) por acción de la raiz pulverizada del barbasco (Lonchocarpus nicou) in vivo.

\begin{tabular}{ccccc}
\hline \multirow{2}{*}{ Concentración $(\mathbf{g} / \mathbf{1 0 0} \mathbf{~ m L})$} & \multicolumn{2}{c}{ Desprendimiento de garrapatas } & Garrapatas \\
\cline { 2 - 4 } & $\mathbf{2 4}$ horas & $\mathbf{4 8}$ horas & $\mathbf{7 2}$ horas & evaluadas \\
\hline T0 $(0,00)$ & $1(2,33 \%)$ & $3(6,98 \%)$ & $4(9,30 \%)$ & 43 \\
T1 $(0,10)$ & $3(10,00 \%)$ & $16(53,33 \%)$ & $25(83,33 \%)$ & 30 \\
T2 $(0,25)$ & $21(40,38 \%)$ & $45(86,54 \%)$ & $49(94,23 \%)$ & 52 \\
T3 $(0,50)$ & $14(32,56 \%)$ & $35(81,40 \%)$ & $43(100,00 \%)$ & 43 \\
T4 $(0,75)$ & $34(54,84 \%)$ & $56(90,32 \%)$ & $62(100,00 \%)$ & 62 \\
\hline Chi cuadrado: $p$ - valor & $<0,0001$ & $<0,0001$ & $<0,0001$ & \\
\hline
\end{tabular}




\section{CONCLUSIÓN}

La raíz pulverizada del barbasco redujo la viabilidad de los huevos y causó el $100 \%$ de mortalidad de larvas y adultos de Rhipicephalus (Boophilus) microplus en concentraciones de 0,50 $\mathrm{g} / 100 \mathrm{~mL}$.

\section{BIBLIOGRAFÍA CITADA}

Bazán, M. 2002. Efecto de Metarhizium anisopliae (Deuteromycotina: Hyphomycetes) en el control de Boophilus microplus Canestrini (Acari: Ixodidae) en ganado bovino estabulado. Tesis MSc. biotecnología. Km 40 autopista ColimaManzanillo, México. Universidad de Colima. 53 p.

Casas, A.; Trigueros, V.; Chavez, V.; Tang, P. y Ruiz, V. 2009. Tratamiento y control de Garrapata Boophilus microplus, a través de la combinación de Fluzuron/fipronil pour on, en bovinos de trópico, Pucallpa, Perú. Tesis Med. Veterinario. Ivita - Pucallpa, Perú. Universidad Nacional Mayor de San Marcos. 11 p.

Espinoza, J. 1994. Efecto de barbasco (Lonchocarpus nicou) en el control de piojos de cuyes, en Tingo María. Tesis Ing. Zootecnista. Tingo maría, Perú. Universidad Nacional Agraria de la Selva. 45 p.

Espinoza, L. 2005. Evaluación de cepas de Beauveria bassiana y de Metarhizium anisopliae en control biológico de Boophilus microplus. Tesis Ing. Agrónomo. Francisco Morazán, Honduras. Escuela Agricola Panamericana, El Zamorano. $29 \mathrm{p}$.

Henrioud, A.N. 2011. Towards sustainable parasites control practices in livestock production with emphasis in Latin America. Veterinary Parasitology 180: 2-11.
Gutiérrez, 2006. Identificación de órganos blanco en garrapatas de la especie Boophilus microplus para anticuerpos-antigarrapata de bovinos inducidos por el inmunógeno Tick-Vac $\mathrm{MK}^{\circledR}$ del laboratorio limor de Colombia S.A mediante métodos de inmunoperoxisadasa. Tesis Microbiología industrial. Bogota. Pontificia Universidad Javeriana. 105 p p . (http://www.javeriana.edu.co/biblos/tesis/cienci as/tesis265.pdf).

Mariños, C.; Castro, J.; Nongrados, D. 2004. Efecto biocida del «barbasco» Lonchocarpus utilis (Smith, 1930) como regulador de larvas de mosquitos. Revista Peruana de Biología, 11 (1): 87-94. (http://www.scielo.org.pe/pdf/rpb/ v11n1/v11n1a11.pdf) Acceso: 14/12/2009.

Reyes, L. 2011. Extracto de rotenona, insecticida natural, organico y biodegradable. [en línea] http://www.sectorproductivo.com.py/agricola/p laguicidas/6277-extracto-de-rotenonainsecticida-natural-organico-y-biodegradable, Revista, 15 de Noviembre 2011.

Silva, G.; Lagunez, J. Rodriguez, R. 2002. Insecticidas vegetales [en línea]: http:// www.monografias.com/trabajos18/insecticidasaturales/insecticidas-naturales.shtml, documento, 10 de Noviembre 2011.

Peralta, R. 2004. Efecto de la raíz molida del kumu (Lonchocarpus sp) en el control de piojos en cuyes (Cavia porcellus). Tesis Ing. Zootecnista. Tingo maría, Perú. Universidad Nacional Agraria de la Selva. 35 p.

Recibido: 10/01/2014

Aceptado para publicación: 30/04/2014 
